\title{
Research Article \\ Dynamics of a Delay-Varying Computer Virus Propagation Model
}

\author{
Jianguo Ren, ${ }^{1}$ Yonghong $\mathrm{Xu}^{2}{ }^{2}$ Yongchang Zhang, ${ }^{1}$ \\ Yongquan Dong, ${ }^{1}$ and Guosheng Hao ${ }^{1}$ \\ ${ }^{1}$ College of Computer, Jiangsu Normal University, Xuzhou 221116, China \\ ${ }^{2}$ College of Bioengineering, Chongqing University, Chongqing 400044, China \\ Correspondence should be addressed to Jianguo Ren, rjgrjgrjgrjg@126.com
}

Received 4 June 2012; Accepted 19 July 2012

Academic Editor: Xiaofan Yang

Copyright (C) 2012 Jianguo Ren et al. This is an open access article distributed under the Creative Commons Attribution License, which permits unrestricted use, distribution, and reproduction in any medium, provided the original work is properly cited.

\begin{abstract}
By considering the varying latency period of computer virus, we propose a novel model for computer virus propagation in network. Under this model, we give the threshold value determining whether or not the virus finally dies out, and study the local stability of the virusfree and virus equilibrium. It is found that the model may undergo a Hopf bifurcation. Next, we use different methods to prove the global asymptotic stability of the equilibria: the virus-free equilibrium by using the direct Lyapunov method and virus equilibrium by using a geometric approach. Finally, some numerical examples are given to support our conclusions.
\end{abstract}

\section{Introduction}

With the advance of computer software andhardware and communication technologies, the number and sort of computer viruses have increased dramatically, which causes huge losses to the human society. Therefore, establishing reasonable computer-virus-propagation models by considering the characteristics of computer virus and, by model analysis, understanding the spread law of the virus over the network, are a currently hot topic of research.

Towards this goal, the classical SIR (susceptible-infected-recovered) model [1, 2], as well as its extensions [3-5], is extended to explore the behavior of computer virus propagation in network. Based on these classical models and by considering the computer virus fixed latent period, Mishra et al. [6,7] proposed delayed SIRS, SEIR computer virus models with a fixed period of temporary immunity, which accounts for the temporary recovery from the infection of virus. In [8], Tan and Han proposed an SIRS computer virus model with fixed latency and temporal immune periods, studied the effect of time delays 
on the stability of the equilibria, and gave some conditions for the equilibria to be locally asymptotically stable for all delays.

Motivated by the previous work, this paper proposes and studies a computer-viruspropagation model with varying latency period, known as the SIRC model. We obtain the threshold value determining whether the virus dies out completely, study the local asymptotic stabilities of the equilibria of the model and it is found that, model may undergo a Hopf bifurcation. Next, we prove the global asymptotic stability of the virus-free equilibrium by using the direct Lyapunov method, prove the global asymptotic stability of the virus equilibrium by using a geometric approach. By introducing varying time delay, the model may truly reflect the virus propagation and hence, the corresponding results may help understand and prevent the spread of computer virus over a computer network.

The remaining materials of this paper are organized this way: Section 2 introduces the mathematical model to be discussed; Section 3 studies the local stability of the virus-free and virus equilibrium of model, respectively, examines the stability switch for a virus equilibrium, and shows that our model may admit a Hopf bifurcation; Section 4 uses different methods to prove the global asymptotic stability of the equilibria. In Section 5, some numerical examples are given to support our conclusions. We end the paper with a brief discussion in Section 6.

\section{Mathematical Model}

Consider the classical SIR computer virus model proposed in [1, 2]

$$
\begin{gathered}
\frac{d S}{d t}=b-\beta S(t) I(t)-\mu S(t), \\
\frac{d I}{d t}=\beta S(t) I(t)-(\gamma+\mu) I(t), \\
\frac{d R}{d t}=\gamma I(t)-\mu R(t) .
\end{gathered}
$$

Here it is assumed that all the computers connected to the network in concern are classified into three categories: susceptible, infected, and recovered computers. Let $S(t), I(t)$, and $R(t)$ denote their corresponding numbers at time $t$. This model involves four positive parameters: $b$ denotes the rate at which external computers are connected to the network, $\gamma$ denotes the recovery rate of infected computers due to the antivirus ability of the network, $\mu$ denotes the rate at which one computer is removed from the network, $\beta$ denotes the rate at which, when having connection to one infected computer, one susceptible computer can become infected. For some variants of this model, see $[1-5,9,10]$.

The computer virus has latent and unpredictable characteristics [11]. A sophisticated computer virus program, when entering into the computer system, does not immediately break out. The longer the latency of a computer virus, the wider its spreading scope will be. On one hand, the computer virus program can not be detected without use of the specialized programs. The virus can stay quietly in the disk or $\mathrm{CD}$ a few days, even years, and when the time comes, it will break out to reproduce, spread, and continue to harm. On the other hand, there is a trigger mechanism within the computer virus, if the trigger conditions are not met, the computer virus does not do any other damage. Only when the trigger conditions are met, can the virus be activated to do some damages. Without loss of reality, the following 
assumptions are made:

(1) The virus in susceptible computer has a latency period. Moreover, this latency period is varying, which can be reflected by the following expression:

$$
\int_{-\infty}^{t} S(\tau) D(t-\tau) d \tau
$$

where $D$ is the delay kernel [12], $\tau$ is the distributed delay, $S(\tau)$ indicates how $S(t)$ is affected by their previous values.

(2) Only when the virus breaks out can the susceptible computers become the infected ones.

We choose a typical class of kernels

$$
D(t-\tau)=\frac{\left\{\sigma^{n+1}(t-\tau)^{n} \exp [-\sigma(t-\tau)]\right\}}{n !}, \quad t \geq 0, n=0,1,2, \ldots,
$$

where $\sigma$ is a positive constant indicating the average delay of the collected information on the virus infection. In this paper, we simply take the weak kernel

$$
D(t-\tau)=\sigma e^{-\sigma(t-\tau)}, \quad \sigma>0,
$$

which implies that the effect of previous events decreases exponentially.

By incorporating these factors into model (2.1), we get the following model:

$$
\begin{gathered}
\frac{d S}{d t}=b-\beta \int_{-\infty}^{t} S(\tau) D(t-\tau) d \tau I(t)-\mu S(t), \\
\frac{d I}{d t}=\beta \int_{-\infty}^{t} S(\tau) D(t-\tau) d \tau I(t)-(\mu+\gamma) I(t), \\
\frac{d R}{d t}=\gamma I(t)-\mu R(t) .
\end{gathered}
$$

We define a new variable

$$
C(t)=\int_{-\infty}^{t} S(\tau) D(t-\tau) d \tau,
$$


which indicates in susceptible computer, the effect of latent virus on infection. Then model (2.5) becomes

$$
\begin{gathered}
\frac{d S}{d t}=b-\beta C(t) I(t)-\mu S(t), \\
\frac{d I}{d t}=\beta C(t) I(t)-(\mu+\gamma) I(t), \\
\frac{d R}{d t}=\gamma I(t)-\mu R(t) \\
\frac{d C}{d t}=\frac{1}{\sigma} S(t)-\frac{1}{\sigma} C(t) .
\end{gathered}
$$

Because $S, I$, and $C$ are independent of variable $R$, this paper focuses on the following model:

$$
\begin{gathered}
\frac{d S}{d t}=b-\beta C(t) I(t)-\mu S(t), \\
\frac{d I}{d t}=\beta C(t) I(t)-(\mu+\gamma) I(t), \\
\frac{d C}{d t}=\frac{1}{\sigma} S(t)-\frac{1}{\sigma} C(t) .
\end{gathered}
$$

Adding the first two equations of model (2.8), we can obtain

$$
\frac{d(S+I)}{d t}=b-\mu(S+I)-\gamma I
$$

Therefore, $S(t)$ and $I(t)$ are bounded, that is, $S+I \leq b / \mu$. From the third equation of model (2.8), we can obtain

$$
\frac{d C}{d t}=\frac{1}{\sigma} S-\frac{1}{\sigma} C \leq \frac{b}{\sigma \mu}-\frac{1}{\sigma} C
$$

It is easy to see that $C(t)$ is bounded, that is, $C \leq b / u$. Thus, the set

$$
\Omega=\left\{(S, I, C) \in R_{+}^{3}, S+I \leq \frac{b}{\mu}, C \leq \frac{b}{\mu}\right\}
$$

is the positively invariant set of model (2.8).

\section{The Equilibria and Local Stability}

This section investigates the equilibria of model (2.8) and their stability. For that purpose, let us introduce the basic reproduction number, which is defined as $R_{0}=b \beta / \mu(\mu+\gamma)$. 
First, model (2.8) has a virus-free equilibrium $E_{0}=(b / \mu, 0, b / \mu)$. The characteristic equation of the corresponding linearized system with respect to $E_{0}$ is

$$
\operatorname{det}\left(\begin{array}{ccc}
-\mu-\lambda & -\frac{\beta b}{\mu} & 0 \\
0 & \frac{\beta b}{\mu}-(\mu+\gamma)-\lambda & 0 \\
\frac{1}{\sigma} & 0 & -\frac{1}{\sigma}-\lambda
\end{array}\right)=0
$$

The three eigenvalues are $-\mu,-1 / \sigma$, and $b \beta / \mu-(\gamma+\mu)$. Thus, we immediately get

Theorem 3.1. Consider model (2.8).

(a) The virus-free equilibrium $E_{0}$ is locally asymptotically stable if $R_{0}<1$.

(b) $E_{0}$ is unstable if $R_{0}>1$.

Next, when $R_{0}>1$, model (2.8) has a positive virus equilibrium $E^{*}=\left(S^{*}, I^{*}, C^{*}\right)$, where

$$
S^{*}=\frac{b}{\mu R_{0}}, \quad I^{*}=\frac{\mu\left(R_{0}-1\right)}{\beta}, \quad C^{*}=\frac{b}{\mu R_{0}} .
$$

The characteristic equation of the corresponding linearized system near $E^{*}$ is

$$
\operatorname{det}\left(\begin{array}{ccc}
-\mu-\lambda & -\beta C^{*} & -\beta I^{*} \\
0 & -\lambda & \beta I^{*} \\
\frac{1}{\sigma} & 0 & -\frac{1}{\sigma}-\lambda
\end{array}\right)=0
$$

which equals

$$
\lambda^{3}+p_{0} \lambda^{2}+p_{1} \lambda+p_{2}=0
$$

where

$$
p_{0}=\mu+\frac{1}{\sigma}, \quad p_{1}=\left(\beta I^{*}+\mu\right) \frac{1}{\sigma}, \quad p_{2}=\frac{1}{\sigma} \beta^{2} C^{*} I^{*} .
$$

A simple calculation gives

$$
\begin{aligned}
p_{0} p_{1}-p_{2} & =\left(\frac{1}{\sigma}+\mu\right)\left(\beta I^{*}+\mu\right) \frac{1}{\sigma}-\beta^{2} C^{*} I^{*} \frac{1}{\sigma} \\
& =\frac{1}{\sigma}\left\{\mu\left(\beta I^{*}+\mu\right)-\beta^{2} C^{*} I^{*}+\left(\beta I^{*}+\mu\right) \frac{1}{\sigma}\right\} .
\end{aligned}
$$

If $p_{0} p_{1}-p_{2}>0$, that is, $\sigma<\sigma^{*}, E^{*}$ is locally asymptotically stable, where $\sigma^{*}=R_{0} /\left(R_{0}-1\right)(\gamma+$ $\mu)-\mu R_{0}$, and $\sigma^{*}>0$ is equivalent to $R_{0}>1+\mu / \gamma$. From the above analysis, we obtain the following Theorem: 
Theorem 3.2. Consider model (2.8). Suppose $R_{0}>1+\mu / \gamma$.

(a) The virus equilibrium $E^{*}$ is locally asymptotically stable if $\sigma<\sigma^{*}$.

(b) $E^{*}$ is unstable if $\sigma>\sigma^{*}$.

Remark 3.3. From the above analysis, we can see that that there exists a stability switch for $E^{*}$ : $E^{*}$ changes its stability when $\sigma$ goes across the critical value $\sigma^{*}$, which may result in a Hopf bifurcation and, hence, can be exploited to find an effective strategy for preventing the spread of computer virus.

Indeed, when $\sigma=\sigma^{*}$, (3.4) has two complex conjugate roots, $\lambda_{1,2}=\alpha(T) \pm i \omega(T)$. It is noted that $\alpha\left(\sigma^{*}\right)=0, \omega\left(\sigma^{*}\right)=\sqrt{p_{1}}>0$, and

$$
\Delta=\left.\frac{d \alpha}{d \sigma}\right|_{\sigma^{*}}=\frac{\sigma^{*} \mu(\gamma+\mu)\left(R_{0}-1\right)-\sigma^{*} \mu^{2} R_{0}-2 \mu R_{0}}{2 \sigma^{* 3}\left(\mu+1 / \sigma^{*}\right)^{2}+2 \sigma^{* 2} \mu R_{0}} .
$$

Let $f\left(R_{0}\right)=\sigma^{*} \mu(\gamma+\mu)\left(R_{0}-1\right)-\sigma^{*} \mu^{2} R_{0}-2 \mu R_{0} \neq 0$, which leads to $\Delta \neq 0$. Thus, we obtain.

Theorem 3.4. If $R_{0}>1+\mu / \gamma$, model (2.8) undergoes a Hopf bifurcation with respect to the virus equilibrium when $\sigma$ goes across the value of $\sigma^{*}$.

\section{Global Stability}

In this section, we will discuss the global stability of the model.

Theorem 4.1. when $R_{0}<1$, the virus-free equilibrium $E_{0}$ is global stability.

Proof. Define

$$
V(S, I, C)=\frac{1}{2} I^{2}
$$

If $R_{0}<1$, then

$$
\begin{aligned}
\dot{V}(S, I, C) & =[\beta C-(\mu+\gamma)] I^{2} \leq\left[\beta \frac{b}{\mu}-(\mu+\gamma)\right] I^{2} \\
& \leq\left[(\mu+\gamma)\left(R_{0}-1\right)\right] I^{2} \leq 0 .
\end{aligned}
$$

Since all the model parameters are positive, it follows that $\dot{V}(S, I, C)<0$ for $R_{0}<1$ with $\dot{V}(S, I, C)=0$ if and only if $I=0$ or $R_{0}=1$. Hence, $V$ is a Lyapunov function on $\Omega$. Thus, $I \rightarrow 0$ as $t \rightarrow \infty$. Using $I=0$ in the first equation of (2.8) shows that $S \rightarrow b / \mu$ as $t \rightarrow \infty$. Therefore, it follows from the Lasalle's invariance principle, that every solution of the model, starting from within $\Omega$, approaches $E_{0}$ as $t \rightarrow \infty$.

In the following, we use the geometrical approach $[13,14]$ to discuss the global stability of virus equilibrium $E^{*}$. First, we give a brief outline of this approach. 

equation:

Let $x \rightarrow f(x) \in R^{n}$ be a $C^{1}$ function for $x$ in an open set $D \in R^{n}$. Consider the following

$$
\dot{x}=f(x) .
$$
made:

Denote by $x\left(t, x_{0}\right)$ the solution with $x\left(t, x_{0}\right)=x_{0}$. Then, the following assumptions are

$\left(\mathrm{H}_{1}\right)$ There exists a compact absorbing set $K \subset D$.

$\left(\mathrm{H}_{2}\right)$ Equation (4.3) has a unique equilibrium $x_{0}$ in $D$.

Let $x \rightarrow p(x)$ be an $\left(\begin{array}{c}n \\ 2\end{array}\right) \times\left(\begin{array}{c}n \\ 2\end{array}\right)$ matrix-valued function that is $C^{1}$ for $x \in D$. Assume that $p^{-1}(x)$ exists and is continuous for $x \in K$, the compact absorbing set. A quantity $\bar{q}_{2}$ is defined as

$$
\bar{q}_{2}=\operatorname{limsupsup}_{x \rightarrow \infty} \frac{1}{x_{0} \in K} \int_{0}^{t} \mu\left(B\left(x\left(s, x_{0}\right)\right)\right) d s,
$$

where

$$
B=p_{f} p^{-1}+p \frac{\partial f^{[2]}}{\partial x} p^{-1}
$$

The matrix $p_{f}$ is obtained by replacing each entry of $p$ by its derivative in the direction of $f$, and $\mu(B)$ is defined by

$$
\mu(B)=\lim _{h \rightarrow 0^{+}} \frac{|I+h B|-1}{h},
$$

which is the Lozinskil measure of $B$ with respect to a vector norm $|\cdot|$ in $R^{N}$.

From the above outline, a theorem can be given as follows:

Theorem 4.2 (see [13]). Assume that $D$ is simply connected, and that the assumptions $\left(H_{1}\right)$ and $\left(\mathrm{H}_{2}\right)$ hold, if $\bar{q}_{2}<0$, then the unique equilibrium $x_{0}$ of (4.3) is globally asymptotically stable.

Now, we discuss the global stability of the virus equilibrium $E^{*}$ of model (2.8).

Model (2.8) has a unique virus equilibrium $E^{*}$ in $\Omega$, hence it satisfies the assumption $\left(\mathrm{H}_{1}\right)$. If $R_{0}>1$, then virus-free equilibrium is not stable, and the solutions of model (2.8) are bounded, which ensure model (2.8) has a compact set in $\Omega$. Therefore, the assumption $\left(\mathrm{H}_{2}\right)$ is met.

The Jacobian matrix of model (2.8) is

$$
J=\left(\begin{array}{ccc}
-\mu & -\beta C & -\beta I \\
0 & \beta C-\mu-\gamma & \beta I \\
-\frac{1}{\sigma} & 0 & -\frac{1}{\sigma}
\end{array}\right)
$$


and its second additive compound matrix is

$$
J^{[2]}=\left(\begin{array}{ccc}
\beta C-2 \mu-\gamma & \beta I & \beta I \\
0 & -\mu-\frac{1}{\sigma} & -\beta C \\
-\frac{1}{\sigma} & 0 & \beta C-(\mu+\gamma)-\frac{1}{\sigma}
\end{array}\right) .
$$

Set the function

$$
P(X)=P(S, I, C)=\operatorname{diag}\left\{\frac{S}{I}, \frac{S}{I}, \frac{S}{I}\right\} .
$$

Then

$$
P_{f} P^{-1}=\operatorname{diag}\left\{\frac{S^{\prime}}{S}-\frac{I^{\prime}}{I}, \frac{S^{\prime}}{S}-\frac{I^{\prime}}{I}, \frac{S^{\prime}}{S}-\frac{I^{\prime}}{I}\right\}
$$

and the matrix $B=P_{f} P^{-1}+P J^{[2]} P^{-1}$ can be written as

$$
B=\left(\begin{array}{ll}
B_{11} & B_{12} \\
B_{21} & B_{22}
\end{array}\right)
$$

where $B_{11}=S^{\prime} / S-I^{\prime} / I+\beta C-2 \mu-r, B_{12}=(\beta I, \beta I), B_{12}=(0,1 / \sigma)^{T}$, and

$$
B_{22}=\left(\begin{array}{cc}
\frac{S^{\prime}}{S}-\frac{I^{\prime}}{I}-\mu-\frac{1}{\sigma} & -\beta C \\
0 & \frac{S^{\prime}}{S}-\frac{I^{\prime}}{I}-\mu-\gamma-\frac{1}{\sigma}+\beta C
\end{array}\right) .
$$

Select the norm in $R^{3}$ as the following:

$$
|u, v, \omega|=\max \{|u|,|v|+|\omega|\}
$$

where $(u, v, w)$ denotes the vector in $R^{3}$, let $\mu$ denote the Lozinskii measure with respect to this norm, then

$$
\mu(B) \leq \sup \left\{g_{1}, g_{2}\right\}=\sup \left\{\mu\left(B_{11}\right)+\left|B_{12}\right|, \mu\left(B_{22}\right)+\left|B_{21}\right|\right\},
$$

where $\left|B_{12}\right|,\left|B_{21}\right|$ are matrix norms with respect to the $L_{1}$ vector norm. Thus,

$$
\begin{gathered}
\mu\left(B_{11}\right)=\frac{S^{\prime}}{S}-\frac{I^{\prime}}{I}+\beta C-2 \mu-r, \\
\left|B_{12}\right|=\beta I \\
\left|B_{21}\right|=\frac{1}{\sigma} .
\end{gathered}
$$


Under the condition of $C \leq \gamma / 2 \beta$,

$$
\mu\left(B_{22}\right)=\frac{S^{\prime}}{S}-\frac{I^{\prime}}{I}-\mu-\frac{1}{\sigma}
$$

Therefore

$$
\begin{gathered}
g_{1}=\frac{S^{\prime}}{S}-\frac{I^{\prime}}{I}+\beta C+\beta I-2 \mu-r \\
g_{2}=\frac{S^{\prime}}{S}-\frac{I^{\prime}}{I}-\mu .
\end{gathered}
$$

According to the second equation of model (2.8), we can obtain

$$
\frac{I^{\prime}}{I}=\beta C-\mu-r
$$

Hence,

$$
\begin{gathered}
g_{1}=\frac{S^{\prime}}{S}-\frac{I^{\prime}}{I}+\beta I+\beta C-2 \mu-\gamma \leq \frac{S^{\prime}}{S}-\left(2 \mu+\gamma-\frac{b \beta}{\mu}\right) \\
g_{2}=\frac{S^{\prime}}{S}-\frac{I^{\prime}}{I}-\mu \leq \frac{S^{\prime}}{S}-\mu-(\mu+\gamma)\left(R_{0}-1\right) \leq \frac{S^{\prime}}{S}-\mu .
\end{gathered}
$$

Therefore,

$$
\mu(B) \leq \frac{S^{\prime}}{S}-\bar{b}
$$

For $t \geq t_{1}$, where $\bar{b}=\min \{\mu, 2 \mu+\gamma-b \beta / \mu\}$.

This leads to

$$
\begin{aligned}
\frac{1}{t} \int_{0}^{t} \mu(B) d s & =\frac{1}{t} \int_{0}^{t_{1}} \mu(B) d s+\frac{1}{t} \int_{t_{1}}^{t} \mu(B) d s \\
& \leq \frac{1}{t} \int_{0}^{t_{1}} \mu(B) d s+\frac{1}{t} \log \frac{S(t)}{S\left(t_{1}\right)}-\bar{b}
\end{aligned}
$$

which implies that $\bar{q}_{2} \leq-\bar{b} / 2<0$.

From the above discussions, we can obtain the following theorem:

Theorem 4.3. When $R_{0}>1$, if then the unique equilibrium $E^{*}$ is globally asymptotically stable. 


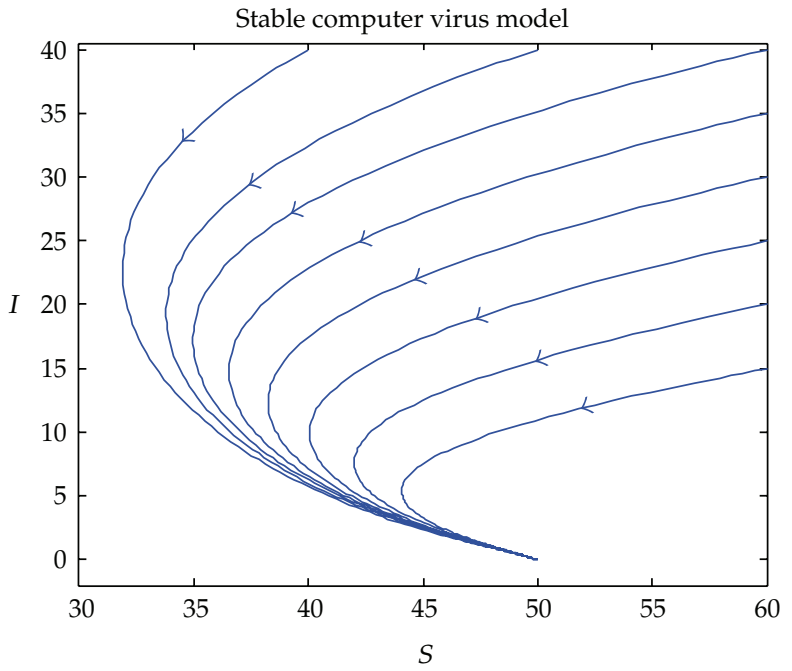

Figure 1: $I$ versus $S$, where $b=20, \beta=0.01, \gamma=0.4, \mu=0.2, \sigma=5$, and $R_{0} \approx 0.83$ satisfy stable condition for the virus-free equilibrium $E_{0}(50.00,0)$.

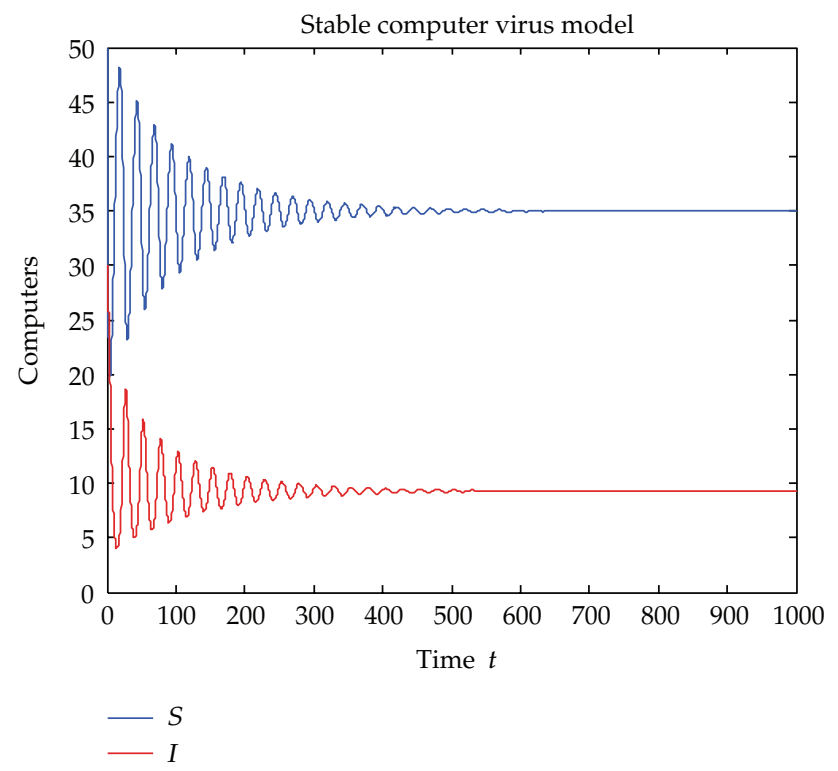

Figure 2: Distribution of computers versus time when $b=10, \beta=0.02, \gamma=0.6, \mu=0.1$, and $\sigma=4$, where $R_{0}=2.86>1$ and $\sigma^{*} \approx 5.91>\sigma$, satisfy the stable condition for the virus equilibrium $E^{*}(35.00,9.29)$.

\section{Numerical Simulations}

In this section, we make some numerical simulations to understand the obtained theorems. Let $b=20, \beta=0.01, \gamma=0.4, \mu=0.2$, and $\sigma=5$, then $R_{0} \approx 0.83<1$. Hence, the virusfree equilibrium $E_{0}(50.00,0)$ is asymptotically stable (see Figure 1 ), that is, the virus would extinguish after a period of time. In contrast, let $b=20, \beta=0.02, \gamma=0.6$, and $\mu=0.1$ yield 


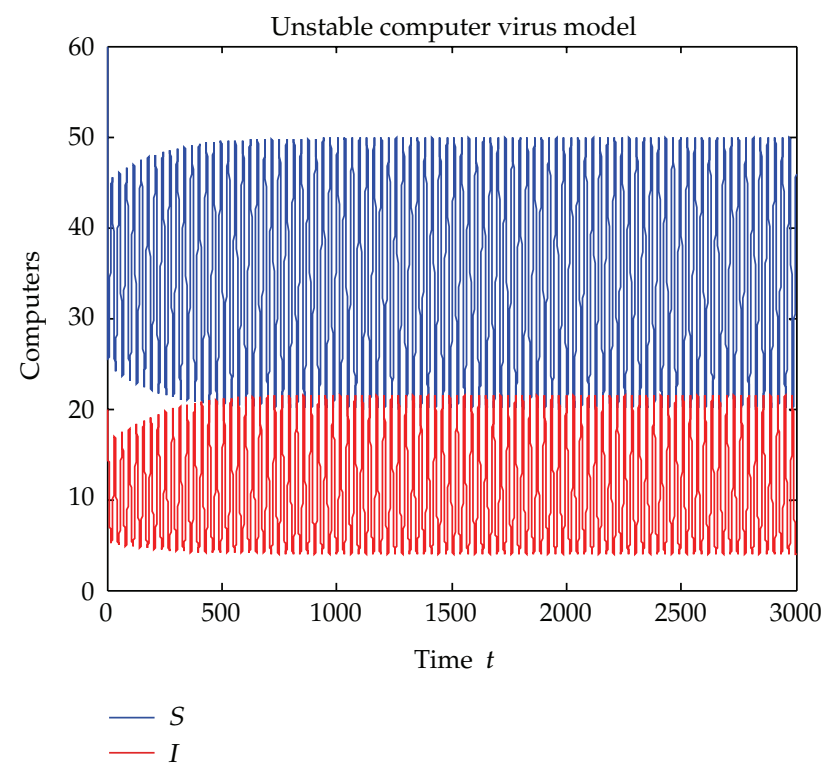

Figure 3: Distribution of computers versus time when $b=20, \beta=0.02, \gamma=0.6, \mu=0.1$, and $\sigma=7.5$, where $R_{0}=2.86>1$ and $\sigma^{*} \approx 5.91<\sigma$ satisfies the unstable condition for the virus equilibrium $E^{*}$.

$\sigma^{*} \approx 5.91$. In this case, when $\sigma=4<\sigma^{*}$ and $\sigma=7.5>\sigma^{*}$, the virus equilibrium $E^{*}(35.00,9.29)$ would become stable (see Figure 2) and unstable (see Figure 3), respectively.

\section{Discussions}

In this paper, by considering varying latency period of computer virus, we propose a model for computer virus propagation in network. First, we give the threshold value $R_{0}$ determining whether the virus extinguishes, and study the local stabilities of the virus-free equilibrium $E_{0}$ and virus equilibrium $E^{*}$ under this model. It is found that $R_{0}$ changes the stability of $E_{0}$ and time delay parameter $\sigma$ changes the stability of $E^{*}$, and that the model may undergo a Hopf bifurcation. Next, we use two different methods to prove the global asymptotic stabilities of the equilibria: the virus-free equilibrium by using the direct Lyapunov method and virus equilibrium by using a geometric approach. Finally, some numerical examples are given to support our conclusions.

\section{Acknowledgments}

The authors wish to thank the anonymous editors and reviewers.

\section{References}

[1] J. C. Wierman and D. J. Marchette, "Modeling computer virus prevalence with a susceptible-infectedsusceptible model with reintroduction," Computational Statistics $\mathcal{E}$ Data Analysis, vol. 45, no. 1, pp. 3-23, 2004.

[2] J. R. C. Piqueira and V. O. Araujo, "A modified epidemiological model for computer viruses," Applied Mathematics and Computation, vol. 213, no. 2, pp. 355-360, 2009. 
[3] B. K. Mishra and N. Jha, "SEIQRS model for the transmission of malicious objects in computer network," Applied Mathematical Modelling, vol. 34, no. 3, pp. 710-715, 2010.

[4] F. Wang, Y. Zhang, C. Wang, J. Ma, and S. Moon, "Stability analysis of a SEIQV epidemic model for rapid spreading worms," Computers and Security, vol. 29, no. 4, pp. 410-418, 2010.

[5] L.-P. Song, Z. Jin, G.-Q. Sun, J. Zhang, and X. Han, "Influence of removable devices on computer worms: dynamic analysis and control strategies," Computers $\mathcal{E}$ Mathematics with Applications, vol. 61, no. 7, pp. 1823-1829, 2011.

[6] B. K. Mishra and D. K. Saini, "SEIRS epidemic model with delay for transmission of malicious objects in computer network," Applied Mathematics and Computation, vol. 188, no. 2, pp. 1476-1482, 2007.

[7] B. K. Mishra and N. Jha, "Fixed period of temporary immunity after run of anti-malicious software on computer nodes," Applied Mathematics and Computation, vol. 190, no. 2, pp. 1207-1212, 2007.

[8] X. Han and Q. Tan, "Dynamical behavior of computer virus on Internet," Applied Mathematics and Computation, vol. 217, no. 6, pp. 2520-2526, 2010.

[9] J. R. C. Piqueira, A. A. de Vasconcelos, C. E. C. J. Gabriel, and V. O. Araujo, “Dynamic models for computer viruses," Computers and Security, vol. 27, no. 7-8, pp. 355-359, 2008.

[10] J. Ren, X. Yang, Q. Zhu, L.-X. Yang, and C. Zhang, "A novel computer virus model and its dynamics," Nonlinear Analysis, vol. 13, no. 1, pp. 376-384, 2012.

[11] Y. B. Kafai, "Understanding virtual epidemics: children's folk conceptions of a computer virus," Journal of Science Education and Technology, vol. 17, no. 6, pp. 523-529, 2008.

[12] S. A. Gourley, "Travelling fronts in the diffusive Nicholson's blowflies equation with distributed delays," Mathematical and Computer Modelling, vol. 32, no. 7-8, pp. 843-853, 2000.

[13] M. Y. Li and J. S. Muldowney, "A geometric approach to global-stability problems," SIAM Journal on Mathematical Analysis, vol. 27, no. 4, pp. 1070-1083, 1996.

[14] M. Y. Li, J. R. Graef, L. Wang, and J. Karsai, "Global dynamics of a SEIR model with varying total population size," Mathematical Biosciences, vol. 160, no. 2, pp. 191-213, 1999. 


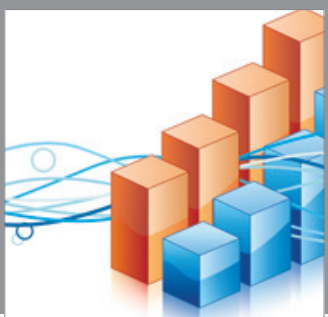

Advances in

Operations Research

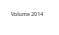

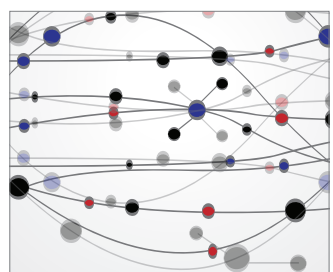

\section{The Scientific} World Journal
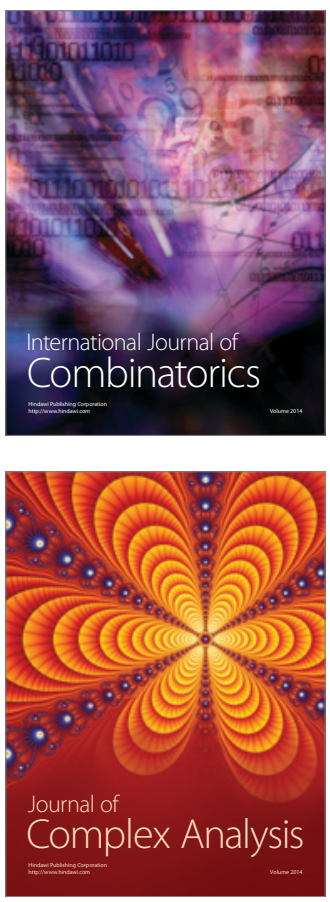

International Journal of

Mathematics and

Mathematical

Sciences
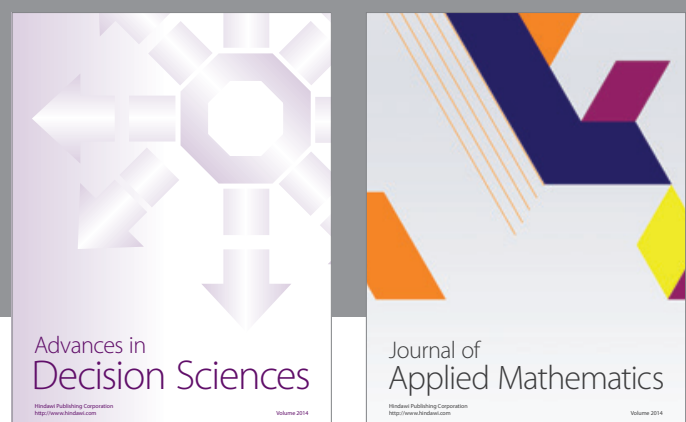

Journal of

Applied Mathematics
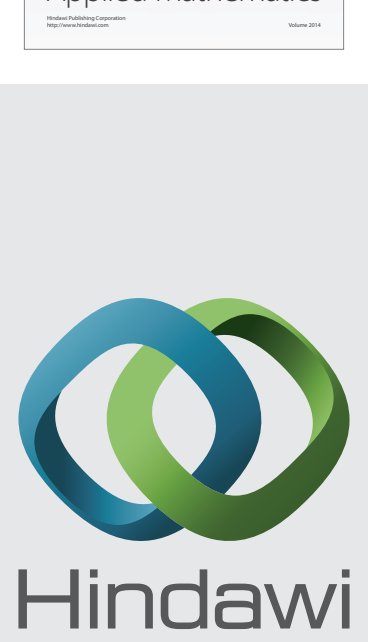

Submit your manuscripts at http://www.hindawi.com
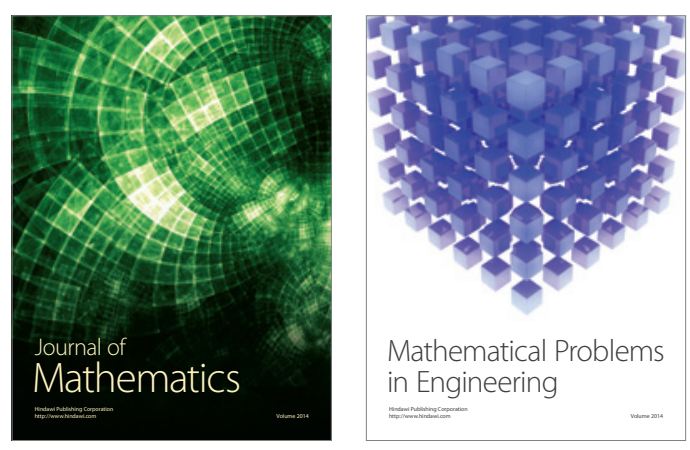

Mathematical Problems in Engineering
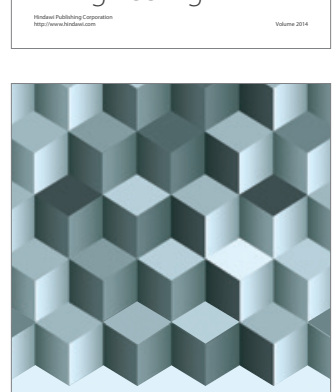

Journal of

Function Spaces
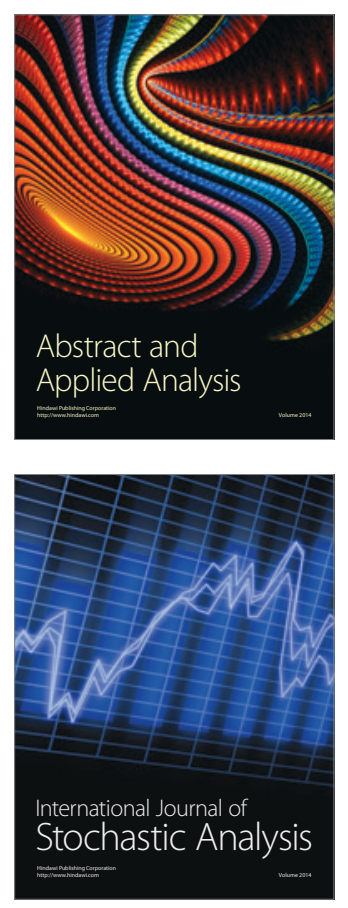

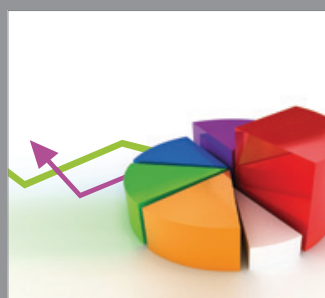

ournal of

Probability and Statistics

Promensencen
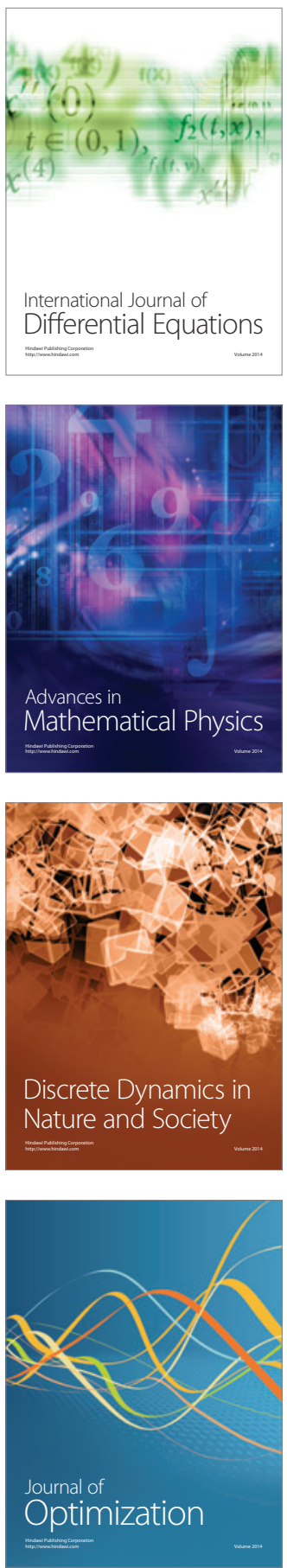